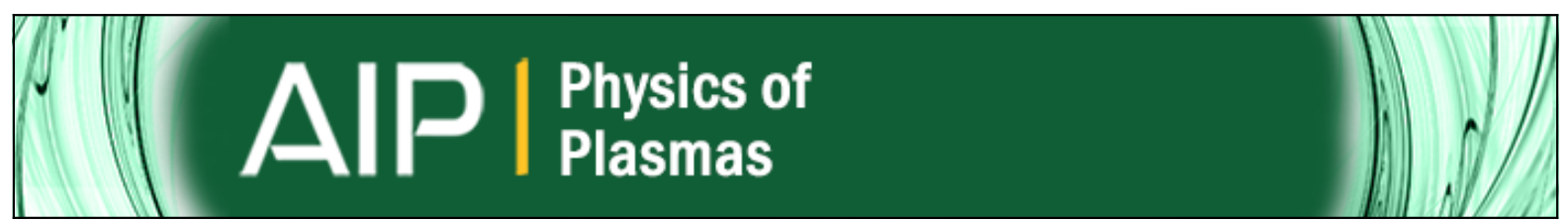

Sub-TeV proton beam generation by ultra-intense laser irradiation of foil-and-gas target

F. L. Zheng, H. Y. Wang, X. Q. Yan, T. Tajima, M. Y. Yu, and X. T. He

Citation: Physics of Plasmas (1994-present) 19, 023111 (2012); doi: 10.1063/1.3684658

View online: http://dx.doi.org/10.1063/1.3684658

View Table of Contents: http://scitation.aip.org/content/aip/journal/pop/19/2?ver=pdfcov

Published by the AIP Publishing

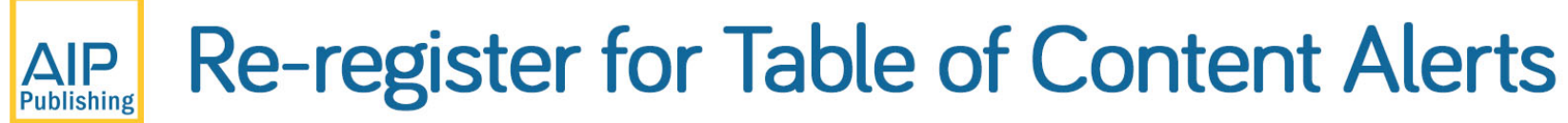




\title{
Sub-TeV proton beam generation by ultra-intense laser irradiation of foil-and-gas target
}

\author{
F. L. Zheng, ${ }^{1,2}$ H. Y. Wang, ${ }^{1}$ X. Q. Yan, ${ }^{1, a)}$ T. Tajima, ${ }^{3}$ M. Y. Yu,,${ }^{4,5}$ and X. T. He ${ }^{1,6, a)}$ \\ ${ }^{1}$ Key Laboratory of HEDP of Ministry of Education, CAPT, State Key Laboratory of Nuclear Physics and \\ Technology, Peking University, Beijing 100871, China \\ ${ }^{2}$ Graduate School, China Academy of Engineering Physics, P.O. Box 8009, Beijing 100088, \\ People's Republic of China \\ ${ }^{3}$ Fakultät für Physik, LMU München, D-85748 Garching, Germany \\ ${ }^{4}$ Institute of Fusion Theory and Simulation, Zhejiang University, Hangzhou 310027, China \\ ${ }^{5}$ Institut für Theoretische Physik I, Ruhr-Universität Bochum, D-44780 Bochum, Germany \\ ${ }^{6}$ Institute of Applied Physics and Computational Mathematics, P. O. Box 8009, Beijing 100088, China
}

(Received 5 September 2011; accepted 11 January 2012; published online 17 February 2012)

\begin{abstract}
A two-phase proton acceleration scheme using an ultra-intense laser pulse irradiating a proton foil with a tenuous heavier-ion plasma behind it is presented. The foil electrons are compressed and pushed out as a thin dense layer by the radiation pressure and propagate in the plasma behind at near the light speed. The protons are in turn accelerated by the resulting space-charge field and also enter the backside plasma, but without the formation of a quasistationary double layer. The electron layer is rapidly weakened by the space-charge field. However, the laser pulse originally behind it now snowplows the backside-plasma electrons and creates an intense electrostatic wakefield. The latter can stably trap and accelerate the pre-accelerated proton layer there for a very long distance and thus to very high energies. The two-phase scheme is verified by particle-in-cell simulations and analytical modeling, which also suggests that a $0.54 \mathrm{TeV}$ proton beam can be obtained with a $10^{23} \mathrm{~W} / \mathrm{cm}^{2}$ laser pulse. (C) 2012 American Institute of Physics. [doi:10.1063/1.3684658]
\end{abstract}

\section{INTRODUCTION}

Proton beams with tens to hundreds $\mathrm{MeV}$ energies are useful for medical imaging, ${ }^{1}$ cancer therapy, ${ }^{2}$ fast ignition in inertial fusion, ${ }^{3}$ conversion of radioactive wastes, ${ }^{4}$ and protons with $\mathrm{TeV}$ energies are relevant to high-energy physics ${ }^{5}$ and astrophysics. ${ }^{6}$ Existing studies have shown that proton beams at the $\mathrm{GeV}$ level can be obtained by radiation pressure acceleration (RPA) using $>10^{22} \mathrm{~W} / \mathrm{cm}^{2}$ lasers. ${ }^{7-12}$ In that scheme the light pressure in the plasma is balanced by the charge-separation field, so that the electron and proton layers co-move as a double-layer. ${ }^{13-20}$ However, because of instability the acceleration length is limited to less than hundreds of micrometers and it is difficult to increase the ion energy. On the other hand, quasi-monoenergetic $\mathrm{GeV}$ electrons can be generated by the laser wakefield acceleration (LWFA) scheme. ${ }^{21-24}$ Recently, Shen et al. ${ }^{25}$ found that a laser pulse at ultra-relativistic intensity can excite a wake field that can capture protons from the underdense plasma and accelerate them to tens of $\mathrm{GeV}$. Furthermore, Yu et al ${ }^{26}$ found that by placing an underdense plasma behind a thin-foil target, $60 \mathrm{GeV}$ protons can be obtained by the fast-moving doublelayer. As a laser pulse propagates in the underdense plasma region, it excites a plasma wakefield with a linear profile which has a negative back part and positive front part. The latter can accelerate protons. Motivated by this, we anticipate that the pre-accelerated protons by RPA can be further accelerated over a long distance by the front positive

\footnotetext{
${ }^{\text {a) }}$ Authors to whom correspondence should be addressed. Electronic addresses: X.Yan@pku.edu.cn and xthe@iapcm.ac.cn.
}

wakefield as long as they are trapped in the acceleration field.

In this paper, we show by particle-in-cell simulation and analytical modeling that with a suitable combination of RPA and LWFA, TeV protons can be generated using a laser of the same intensity. In the proposed two-phase acceleration scheme, an ultra-intense laser irradiates a foil target with a backside plasma, as shown in Fig. 1(a). The foil thickness $d$ satisfies,

$$
l_{0}<d<D,
$$

where $l_{0}, n_{e}, n_{c}$, and $\lambda_{l}$ are the plasma skin depth, plasma density, critical plasma density, and laser wavelength, respectively, $D=\frac{1}{2 \pi} \frac{n_{c}}{n_{e}} a_{0} \lambda_{l}$ is the initial thickness of the foil, ${ }^{13-20}$ $a_{0}=e A / m_{e} \omega_{l} c, A$ is the laser vector potential, and $c$ is the speed of light. The condition means that the light pressure exerted on the electrons is larger than the space-charge force, i.e., the condition for double-layer formation is not satisfied. The RP quickly compresses and pushes the electrons to the rear of the foil, as shown in Fig. 2(a). The electron layer can reach a density far greater than $\gamma n_{c}$, where $\gamma=\left(1+a_{0}^{2}\right)^{1 / 2}$. Meanwhile, the protons in the foil are accelerated by the space-charge field. The electron-layer in rear of the foil is pushed out of the thin foil by the RP just before quasisteady electron-ion double layers can form. The electron layer thus propagates in the underdense gas while being continuously accelerated by the laser pulse like a light-sail. Due to the snowplow effect, the layer is enhanced by the electrons from the backside plasma ${ }^{27}$ and a wake field is formed. The large electric field of the latter in turn accelerates the pre-accelerated (by RPA) proton layer (PL) from the foil, starting the stable 
a)

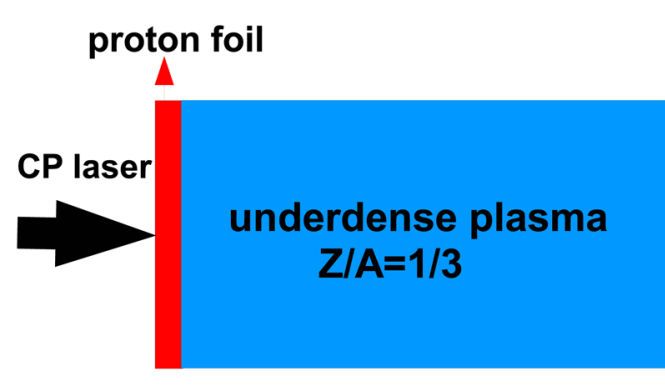

b)

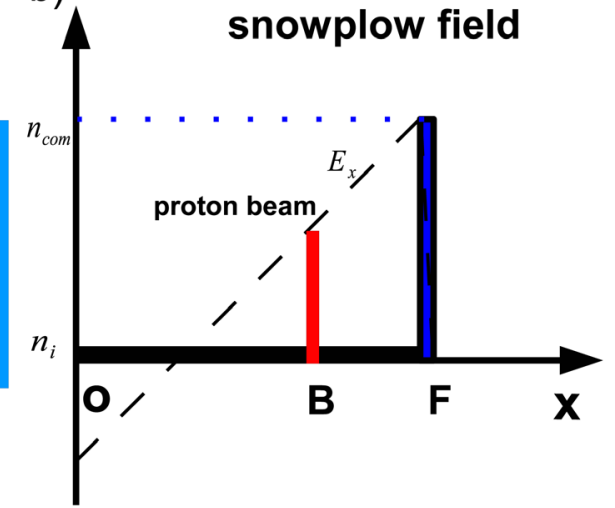

FIG. 1. (Color online) (a) The two-stage acceleration scheme. The initial density of the hydrogen foil is $n_{0} / n_{c}=20$, the thickness is $d=0.5 \lambda_{l}$, the density of the tenuous backside plasma is $n_{e} / n_{c}=0.01$, its length is $12000 \lambda_{l}$. (b) Sketch of the snowplow process, the dynamic density of the ions is $n_{i}$ and the electron density in the snowplow layer is $n_{\text {com. }}$. The position $B$ indicates the laser pulse front and $A$ is an arbitrary point in the snowplow region. long-distance acceleration in the backside plasma, as shown in Fig. 1(b). This is possible because the heavy ions of the backside plasma remain almost unaffected by the wake electric field. The two-phase scheme therefore differs significantly from the existing acceleration schemes invoking the doublelayer, where $d \sim D$ and the accelerated protons can quickly overtake the double layers, so that their energy gain is limited because of decoupling of the protons from the accelerating field. $^{26}$

\section{THE TWO-PHASE ACCELERATION SCHEME}

It is instructive to first discuss our one-dimensional analytical model, which extends that of Esarey ${ }^{29}$ for ultrarelativistic-laser driven plasma wakefields. The evolution of such a wakefield is characterized by (i) lengthening of the wakefield size $l_{s}=\left(\gamma n_{c} / n_{e}\right)^{1 / 2} \lambda_{l}$, and (ii) enhancement of the wavebreaking field $E_{B}=3 \pi\left(\gamma n_{e} / n_{c}\right)^{1 / 2} E_{0}$, where $E_{0}=m_{e} \omega_{l} c / e$. That is, both $l_{s}$ and $E_{B}$ scale with $\gamma^{1 / 2}$. When $d$ satisfies Eq. (1), the pre-accelerated PL can be captured by the wakefield in the underdense plasma and be stably accelerated in the so-called snowplow regime of LWFA over a long distance. The wakefield is composed of two components, which are generated by the compressed dense electron layer and back-side plasma ions. Accordingly, using Poisson equation we can estimate the electrostatic field $E_{A}$ at an point $x_{A}$ in the snowplow region, (see Fig. 1(b)). The contribution of the compressed dense electron layer at position $x_{A}$ is close to $E_{A}^{\prime}=4 \pi e n_{e} l_{s} / 2$ and the contribution of backside plasma ions is $E_{A}^{\prime \prime}=4 \pi e n_{i} x_{A}-4 \pi e n_{i}\left(l_{s}-x_{A}\right)$. The longitudinal electrostatic field at $x_{A}$ is thus $E_{A}=E_{A}^{\prime}+E_{A}^{\prime \prime}=4 \pi e n_{e}$ $\left(2 x_{A}-l_{S} / 2\right)$. Thus the maximum snowplow field at the laser front $x_{B}$ is

$$
E_{B}=3 \pi\left(\gamma n_{e} / n_{c}\right)^{1 / 2} E_{0}
$$

The captured pre-accelerated foil PL is at first slower than the laser pulse as well as the electron layer because of the (a)

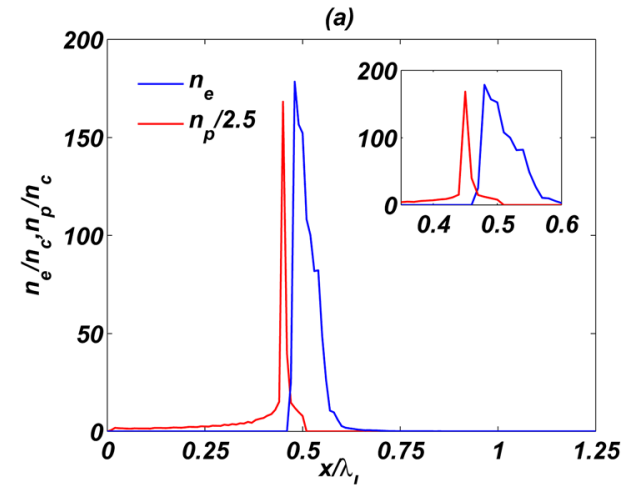

(c)

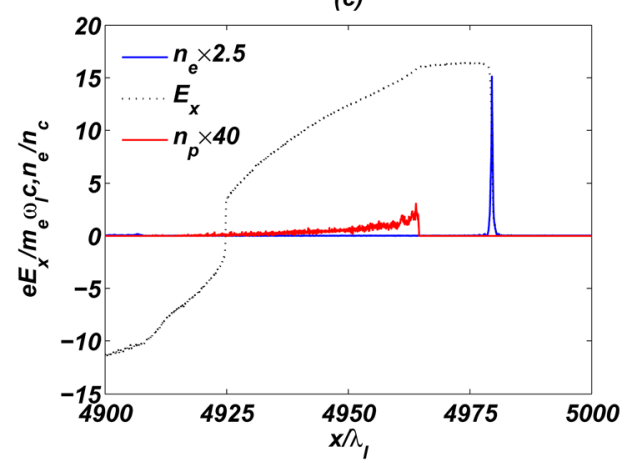

(b)

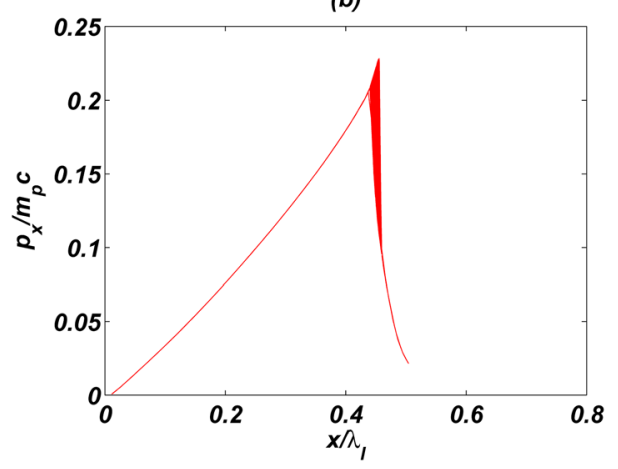

(d)

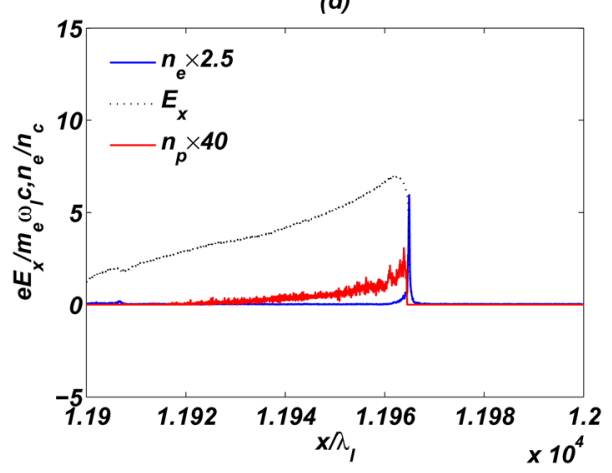

FIG. 2. (Color online) (a) Electron density $n_{e} / n_{c}$, proton density $n_{p} / n_{c}$, and the distance between the electron and PLs (see inset), and (b) longitudinal proton phase space $p_{x} / m_{p} c$ at $t=5.8 T_{l}$, where $m_{p}$ is proton mass. The longitudinal electrostatic field $e E_{x} / m_{e} \omega_{l} c$ (dashed line) in the snowplow regime at (c) $t=5000 T_{l}$ and (d) $t=12000 T_{l}$. The initial plasma parameters are given in Fig. 1. 
condition (1), which, however, weakens rapidly as the electrons there are pulled back by the space-charge force. ${ }^{28}$ On the other hand, the PL is stably accelerated in the wake field and can achieve very high energy after some distance. It is convenient to introduce a length, namely $l_{\text {inj }}$, between the PL and the laser-pulse front. Once the PL enters into the wakefield, its maximum achievable energy depends on the laser depletion length and proton decoupling (from the accelerating field) length. In the present snowplow regime, the laser strongly interacts with only the compressed electron layer because its rear part is in a region void of electrons. As a result, the energy loss is localized near the laser front, causing the front part of the laser to etch backward as it propagates. Pump depletion is thus the limiting mechanism for the survival of the wakefield. The laser depletion length $L_{p d}$ is given by ${ }^{29}: E_{x}^{2} L_{p d} \sim E_{l}^{2} L_{l}$, where $E_{x}$ is the longitudinal electrostatic field in the snowplow regime and $L_{l}$ is the length of laser pulse. Assuming that the group velocity of the laser pulse is close to $c$, we have

$$
L_{p d} \sim L_{l} c / v_{\text {etch }},
$$

where $v_{\text {etch }}$ is the etching velocity of the laser pulse and it reads $v_{\text {etch }} \simeq E_{B}^{2} c / E_{l}^{2}=9 \pi^{2} n_{e} c / a_{0} n_{c}$. Clearly it is proportional to $n_{e} / a_{0}$. Since the electron layer is formed by laser compression, its speed is from the beginning the same as that of the front of the laser pulse, namely $v_{f}=v_{g}-v_{\text {etch }}$, where $v_{g}$ is the laser group velocity.

The decoupling length of the PL is approximately $L_{d p} \sim l_{\text {inj }} v_{p} /\left(v_{p}-v_{f}\right)=l_{\text {inj }} v_{p} /\left[v_{p}-\left(v_{g}-v_{\text {etch }}\right)\right]$, where $v_{p}$ is the proton velocity and $l_{\text {inj }} \sim l_{s} / 4$ from the simulation result. When $v_{g}$ and $v_{p}$ are both near the light speed, so that $v_{\text {etch }} \gg v_{p}-v_{g}$, the proton decoupling length becomes

$$
L_{d p} \sim \frac{1}{36 \pi^{2}}\left(\frac{a_{0} n_{c}}{n_{e}}\right)^{3 / 2} \lambda_{l} .
$$

For $L_{l}>\left(a_{0} n_{c} / n_{e}\right)^{1 / 2} \lambda_{l} / 4$, the laser pump depletion length is larger than the decoupling length. So that the maximum energy the PL can achieve is

$$
W_{\max } \sim\left(n_{c} / 6 n_{e}\right) m_{e} c^{2} a_{0}^{2},
$$

which shows that the present two-phase scheme offers efficient proton acceleration. The acceleration length in the snowplow regime is proportional to $a_{0}^{3 / 2}$ and maximum proton energy scales with $a_{0}^{2}$. This scaling law indicates that a circularly polarized $(\mathrm{CP}) 10^{23} \mathrm{~W} / \mathrm{cm}^{2} 116$ fs laser pulse can generate a quasi-monoenergetic proton bunch with sub-TeV energy. In the following, we shall confirm this conclusion using PIC simulations, which amounts to following the trajectories of charged particles in self-consistent electromagnetic fields computed on a fixed mesh.

\section{SIMULATION RESULTS AND DISCUSSION}

For the simulation, we shall use the fully relativistic PIC code KLAP. ${ }^{13,30}$ The $\lambda_{l}=1 \mu \mathrm{m}$ CP laser pulse has an axially trapezoidal profile with a $15 \lambda_{l}$ flat top and a $20 \lambda_{l}$ ramp on the rising side. The ramp is given by $a=a_{0} \sin ^{2}\left(\pi t / 40 T_{l}\right)$ with $a_{0}=250$, where $T_{l}$ is the laser period. At $t=0$ it is normally incident from the left on a uniform, fully ionized hydrogen foil of thickness $d=0.5 \mu \mathrm{m}$ and normalized density $N=n_{0} /$ $n_{c}=20$. Behind the hydrogen foil is a tenuous heavy-ionplasma of density $n_{e}=0.01 n_{c}$ and charge-to-mass ratio $1 / 3$. Here the simulation box is length $12000 \lambda_{l}$ in the $x$ direction. The spatial resolution is 40 cells per $\lambda_{l}$. Each cell is filled with 20000 macroparticles for the foil plasma and 10 macroparticles for the tenuous backside plasma. The initial electron and ion temperatures are both $1 \mathrm{keV}$.

We first consider the 1D problem, as in the analysis in the Sec. II. As shown in Fig. 2(a), at $t=5.8 T_{l}$, or the end of the laser-foil interaction when space-charge accelerated and compressed PL enter the backside plasma, the electron layer ahead of the laser pulse runs faster than the PL at near the light speed. No quasisteady double-layer can form because the RP force exerted on the electrons is larger than the spacecharge force. Fig. 2(b) shows that in this stage the maximum energy of the PL is about $20 \mathrm{MeV}$ and the speed is about $0.2 c$. The distance between the electron and proton layers continues to increase, as shown in the inset of Fig. 2(a). The laser pulse, now propagating in the backside plasma, snowplows the electrons in front of it and excites an intense electrostatic plasma wave in its wake, thus starting the second stage of proton acceleration in the present scheme.

Snapshots of the electron density, proton density, and electrostatic field are given in Figs. 2(c) and 2(d). We can see that the PL is continuously accelerated until the laser pulse is almost completely depleted at $t=12000 T_{l}$. At that time, the
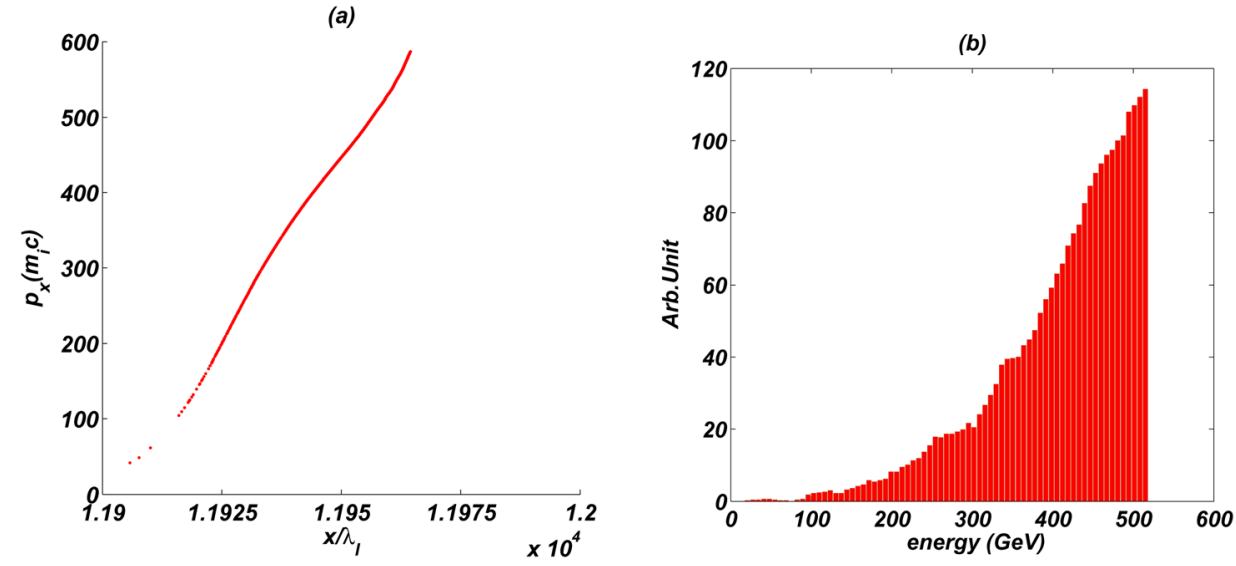

FIG. 3. (Color online) Simulation results at $t=12000 T_{l}$. (a) Proton phase space $p_{x} / m_{p} c$ versus $x$, and (b) energy spectrum of the trapped protons. 
(a)

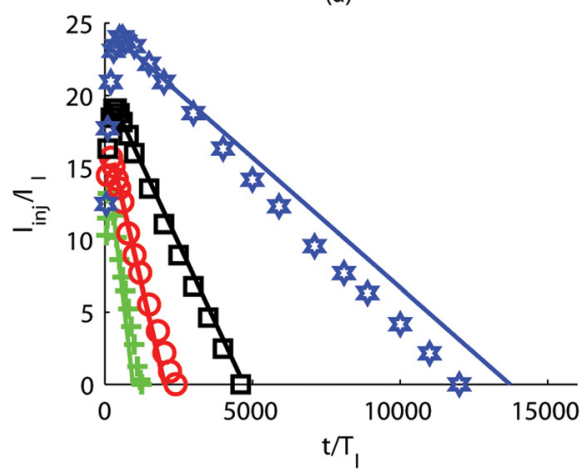

(c)

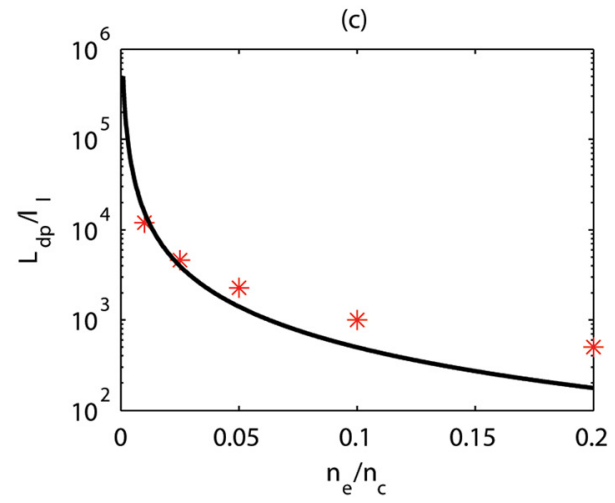

(b)

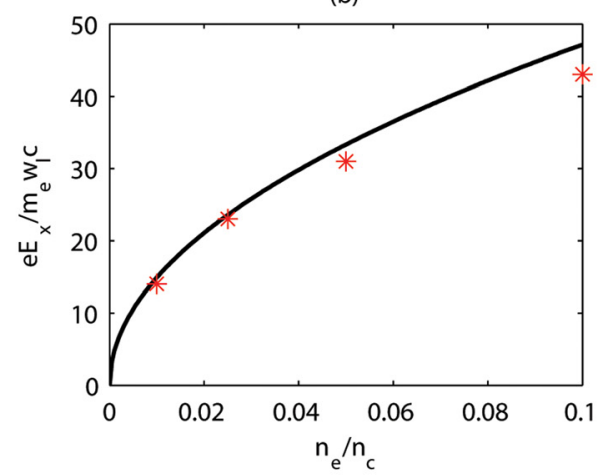

(d)

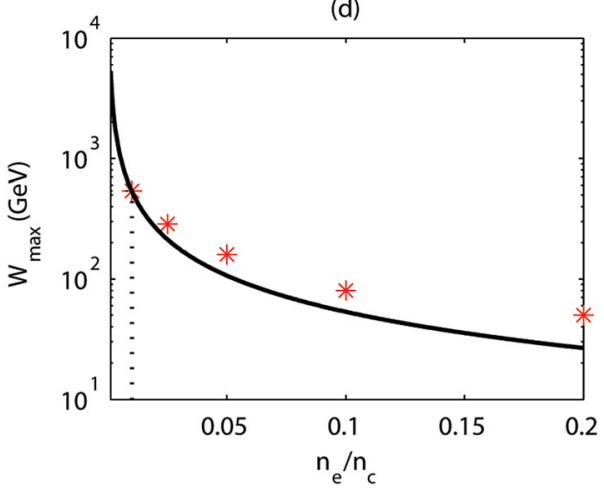

FIG. 4. (Color online) (a) Distance between proton beam and the laser pulse front versus time for different gas densities, where stars, diamonds, crosses, and squares correspond to the gas densities $0.01 n_{c}, 0.025 n_{c}, 0.05 n_{c}$, and $0.1 n_{c}$, respectively, (b) longitudinal electrostatic field $e E_{x} / m_{e} \omega_{l} c$, (c) decoupling length, (d) maximum proton energy. Here the theoretical curves are given as solid lines and the simulation results are shown as stars, diamonds, crosses, and squares.
PL approaches the laser front, leading to substantial reduction of the electrostatic field, as shown in Fig. 2(d). According to our analytical model, we have $E_{x}=14.9 E_{0}, l_{p d}=11125 \lambda_{l}$, $W_{\text {max }}=532 \mathrm{GeV}, v_{\text {etch }}=0.0036 c$, and $v_{f}=0.9964 c$. These results agree well with that from the simulation. Fig. 3 shows that the energy spread (FWHM) of the beam is less than $20 \%$. The maximum proton energy is $540 \mathrm{GeV}$ and the averaged energy of the PL is $437 \mathrm{GeV}$. These are about 8 times that of the corresponding double-layer accelerating scheme. ${ }^{26}$ About $1.72 \%$ of the protons are located in this energy window. The $\mathrm{PL}$ is compressed in the phase space and the length of the final quasi-monoenergetic layer is shorter than $100 \mu \mathrm{m}$. Such a layer can be used to excite a plasma wakefield that can in turn accelerate the plasma electrons to hundreds of $\mathrm{GeV} .^{31}$

As can be seen at the left edge of Fig. 4(a), the distance between the proton beam and the laser front increases up to (a)

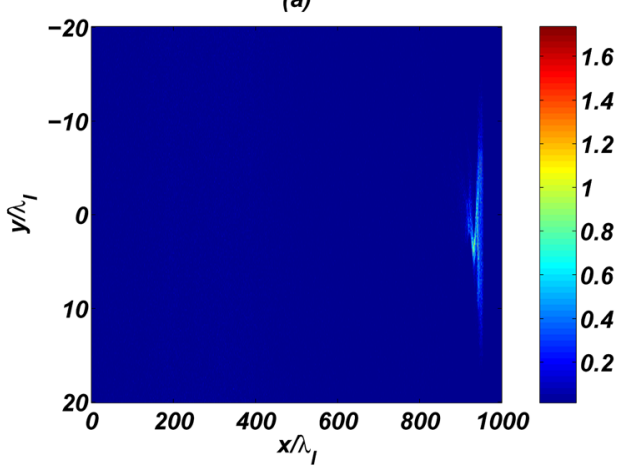

(c)

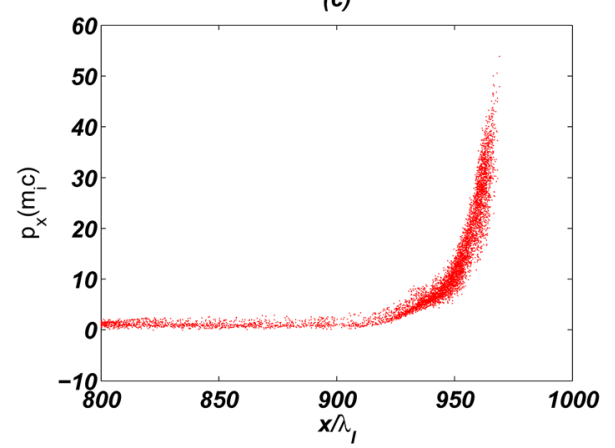

(b)
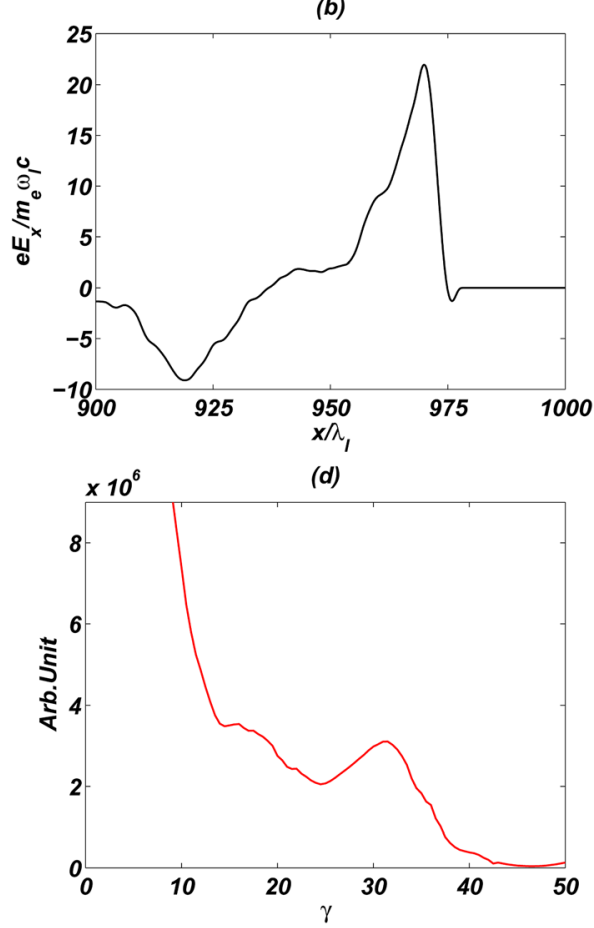

FIG. 5. (Color online) 2D simulation results at $t=990 T_{l}$. (a) Electron density profile, (b) electrostatic field on the axis, (c) proton phase space, and (d) proton spectrum. 
a maximum (corresponding to $l_{\text {inj }}$ ). This distance then decreases due to the erosion of the laser pulse. The slopes of the curves correspond to the etching velocity of the laser pulse for the different backside-plasma densities. The maximum longitudinal-electrostatic field, decoupling length, and maximum proton energy versus the backside-plasma density are shown in Figs. 4(b)-4(d). We see that the maximum energy of the proton beam decreases with the plasma density. To the left of the dotted line (at $n_{e}<0.01 n_{c}$ ), the pulse depletion length is shorter than the decoupling length. Accordingly, to increase the proton energy, one can increase the laser intensity and reduce the backside-plasma density, so that both the decoupling length and pump depletion length are increased.

It is difficult to perform full scale 2D simulations (for obtaining sub-TeV protons) with our computing resources. We therefore consider a narrow simulation box $40 \times 1000 \lambda_{l}^{2}$ with resolution of 5 cells $/ \lambda_{l}$ in the $y$ plane and 20 cells $/ \lambda_{l}$ in the $x$ plane. Each cell contains 4 particles for the backside plasma and 400 particles for the foil plasma. The foil parameters are the same as that in the $1 \mathrm{D}$ simulations, but the backsideplasma density is $0.2 n_{c}$. The super-Gaussian laser pulse is given by $I=I_{0} \exp \left\{-\left(r / r_{0}\right)^{4}-\left[\left(t-t_{0}\right) / \tau\right]^{8}\right\}$, where $r_{0}=10 \mu \mathrm{m}$, $t_{0}=55$ fs, and $\tau=55$ fs. Fig. 5 shows that the snowplowed electron layer, the electrostatic field, and the phase space are similar to the $1 \mathrm{D}$ results. The maximum proton energy of about $50 \mathrm{GeV}$ also agrees with the $1 \mathrm{D}$ simulation. Since the protons gain energy mainly from the electrostatic wake field, ${ }^{26}$ the return current and magnetic field that appear in the 2D simulation do not affect proton acceleration in the snowplow regime. We also note that depreciation of the electron layer is compensated by the laser-snowplowed electrons from the backside plasma, so that the electron layer still exists at the end of our simulations. To suppress transverse instabilities such as Rayleigh-Taylor instability associated with laser-foil interactions, ${ }^{13-20}$ an ultraintense laser pulse and an ultra-thin foil are needed (just as these in the present acceleration scheme), since the instability growth becomes much slower due to relativistic effect in a moving frame. In fact, in the 2D case studied here, there is no evidence of these instabilities. Furthermore, as the pre-accelerated protons are detached from the initial foil, this transverse instability does not appear, since the main acceleration occurs in the stable LWFA stage, where the laser pulse interacts with a very low density underdense plasma.

\section{SUMMARY}

In summary, a two-phase acceleration scheme using a foil and gas target driven by the ultra-intense laser pulse is presented. A 1D model that predicts the proton decoupling length, pump depletion length, and the maximum proton energy is given. The results are verified by $1 \mathrm{D}$ as well as 2D PIC simulations. It is shown that sub-TeV quasi-monoenergetic proton bunches can be generated by a centimeter-scale accelerator with a laser pulse of intensity $10^{23} \mathrm{~W} / \mathrm{cm}^{2}$ and duration $116 \mathrm{fs}$. The resulting proton beam length is shorter than $100 \mu \mathrm{m}$.

\section{ACKNOWLEDGMENTS}

We thank G. Mourou, C. Y. Zheng, H. Zhang, B. Liu, and H. C. Wu for useful discussions and help. This work was supported by the National Nature Science Foundation of China (Grants 10935002, 10974022, 11025523, 11175026, 11175029, 11105009) and the National Basic Research Program of China (Grant 2012CB80111). XQY would like to thank the Alexander von Humboldt Foundation for support.

${ }^{1}$ M. Borghesi, A. Schiavi, D. H. Campbell, M. G. Haines, O. Willi, A. J. Mackinnon, P. Patel, M. Galimberti, and L. A. Gizzi, Rev. Sci. Instrum. 74, 1688 (2003).

${ }^{2}$ S. V. Bulanov, T. Zh. Esirkepovb, V. S. Khoroshkovc, A. V. Kuznetsovb, and F. Pegoraro, Phys. Lett. A 299, 240 (2002); T. Esirkepov, S. V. Bulanov, K. Nishihara, T. Tajima, F. Pegoraro, V. S. Khoroshkov, K. Mima, H. Daido, Y. Kato, Y. Kitagawa, K. Nagai, and S. Sakabe, Phys. Rev. Lett. 89, 175003 (2002).

${ }^{3}$ M. Roth, T. E. Cowan, M. H. Key, S. P. Hatchett, C. Brown, W. Fountain, J. Johnson, D. M. Pennington, R. A. Snavely, S. C. Wilks, K. Yasuike, H. Ruhl, F. Pegoraro, S. V. Bulanov, E. M. Campbell, M. D. Perry, and H. Powell, Phys. Rev. Lett. 86, 436 (2001).

${ }^{4}$ K. W. D. Ledingham, P. McKenna, T. McCanny, S. Shimizu, J. M. Yang, L. Robson, J. Zweit, J. M. Gillies, J. Bailey, G. N. Chimon, R. J. Clarke, D. Neely, P. A. Norreys, J. L. Collier, R. P. Singhal, M. S. Wei, S. P. D. Mangles, P. Nilson, K. Krushelnick, and M. Zepf, J. Phys. D 36, L79 (2003).

5 J. W. Shearer, J. Garrison, J. Wong, and J. E. Swain, Phys. Rev. A 8, 1582 (1973).

${ }^{6}$ S.C. Wilks, A. B. Langdon, T. E. Cowan, M. Roth, M. Singh, S. Hatchett, M. H. Key, D. Pennington, A. MacKinnon, and R. A. Snavely, Phys Plasma 8, 542 (2001).

${ }^{7}$ A. Macchi, F. Cattani, T. V. Liseykina, and F. Cornolti, Phys. Rev. Lett. 94, 165003 (2005).

${ }^{8}$ X. Zhang, B. F. Shen, X. M. Li, Z. Y. Jin, F. C. Wang, and M. Wen, Phys. Plasmas 14, 123108 (2007).

${ }^{9}$ X. Q. Yan, C. Lin, Z. M. Sheng, Z. Y. Guo, B. C. Liu, Y. R. Lu, J. X. Fang, and J. E. Chen, Phys. Rev. Lett. 100, 135003 (2008).

${ }^{10}$ A. P. L. Robinson, M. Zepf, S. Kar, R. G. Evans, and C. Bellei, New J. Phys. 10, 013021 (2008).

${ }^{11}$ C. S. Liu, V. K. Tripathi, and X. Shao, AIP Conf. Proc. 1061, 246 (2008).

${ }^{12}$ A. Henig, S. Steinke, M. Schnüer, T. Sokollik, R. Hörlein, D. Kiefer, D. Jung, J. Schreiber, B. M. Hegelich, X. Q. Yan, J. Meyer-ter-Vehn, T. Tajima, P.V. Nickles, W. Sandner, and D. Habs, Phys. Rev. Lett. 103, 245003 (2009).

${ }^{13}$ X. Q. Yan, H. C. Wu, Z. M. Sheng, J. E. Chen, and J. Meyer-ter-Vehn, Phys. Rev. Lett. 103, 135001 (2009).

${ }^{14}$ T. Esirkepov, M. Borghesi, S. V. Bulanov, G. Mourou, and T. Tajima, Phys. Rev. Lett. 92, 175003 (2004).

${ }^{15}$ B. Qiao, M. Zepf, M. Borghesi, and M. Geissler, Phys. Rev. Lett. 102, 145002 (2009).

${ }^{16}$ M. Chen, A. Pukhov, and T. P. Yu, Phys. Rev. Lett. 103, 024801 (2009).

${ }^{17}$ F. Pegoraro and S. V. Bulanov, Phys. Rev. Lett. 99, 065002 (2007).

${ }^{18}$ L. Yin, B. J. Albright, B. M. Hegelich, K. J. Browers, K. A. Flippo, T. J. T. Kwan, and J. C. Fernandez, Phys. Plasmas 14, 056706 (2007).

${ }^{19}$ Z. M. Zhang, X. T. He, Z. M. Sheng, and M. Y. Yu, Phys. Plasmas 17, 043110 (2010).

${ }^{20}$ V. K. Tripathi, C. S. Liu, X. Shao, B. Eliasson, and R.Z. Sagdeev, Plasma Phys. Controlled Fusion 51, 024014 (2009).

${ }^{21}$ T. Tajima and J. M. Dawson, Phys. Rev. Lett. 43, 267 (1979).

${ }^{22}$ A. Pukhov and J. Meyer-ter-Vehn, Appl. Phys. B 74, 355 (2002).

${ }^{23}$ W. P. Leemans, B. Nagler, A. J. Gonsalves, C. Toth, K. Nakamura, C. G. R. Geddes, E. Esarey, C. Schroeder, and S. M. Hooker, Nat. Phys. 2, 696 (2006).

${ }^{24}$ W. Lu, C. Huang, M. Zhou and M. Tzoufras, Phys. Plasmas 13, 056709 (2006).

${ }^{25}$ B. F. Shen, X. Zhang, Z. M. Sheng, M. Y. Yu, and J. Cary, Phys. Rev. ST Accel. Beams 12, 121301 (2009).

${ }^{26}$ L. L. Yu, H. Xu, W. M. Wang, Z. M. Sheng, B. F. Shen, W. Yu, and J. Zhang, New J. Phys. 12, 045021 (2010).

${ }^{27}$ M. Ashour-Abdalla, J. N. Leboeuf, T. Tajima, J. M. Dawson, and C. F. Kennel, Phys. Rev. A 23, 1906 (1981).

${ }^{28}$ X. Zhang, B. F. Shen, L. L. Ji, F. C. Wang, M. Wen, W. P. Wang, J. C Xu, and Y. H. Yu, Phys. Plasmas 17, 123102 (2010).

${ }^{29}$ E. Esarey and M. Pilloff, Phys. Plasmas 2, 1432 (1995).

${ }^{30}$ Z. M. Sheng, K. Mima, Y. Sentoku, K. Nishihara, and J. Zhang, Phys. Plasmas 9, 3147 (2002).

${ }^{31}$ A. Caldwell, K. Lotov, A. Pukhov, and F. Simon, Nat. Phys. 5, 363 (2009). 